\title{
Fracturas Mandibulares Conminutadas por Impacto de Proyectil Balístico: Resultados Clínicos de Tratamiento con Método de Fijación Alternativo
}

\author{
Mandibular Fractures Comminuted by Ballistic Projectile Impact: \\ Clinical Results of Treatment with Alternative Fixation Method
}

\author{
Marcelo Mardones M. ${ }^{1,2,3}$; Coral Torres M. ${ }^{1}$; Renato Gunckel M. ${ }^{1,2,3}$ \& Rodrigo Bravo A. ${ }^{1,2,3}$
}

\begin{abstract}
MARDONES, M. M.; TORRES, M. C.; GUNCKEL, M. R. \& BRAVO, A. R. Fracturas mandibulares conminutadas por impacto de proyectil balístico: Resultados clínicos de tratamiento con método de fijación alternativo. Int. J. Odontostomat., 15(2):363-369, 2021.

RESUMEN: Las fracturas mandibulares conminutadas por impacto de proyectil balístico son un desafío terapéutico debido a la presencia de múltiples fragmentos óseos y el compromiso a nivel de periostio, plano muscular y mucoso. Su tratamiento inicial requiere de una estabilización de las urgencias médico - quirúrgicas y posteriormente tratar los segmentos óseos fracturados. La fijación externa con tutores mandibulares permite estabilizar los segmentos mandibulares de forma cerrada manteniendo así la viabilidad de los fragmentos sin interrumpir su suministro de sangre. El objetivo de este trabajo es presentar los resultados post operatorios obtenidos en tres casos clínicos de pacientes con diagnóstico de fractura mandibular conminutada por trauma por impacto de proyectil balístico, tratados por un método alternativo a los tutores de fijación externa convencional.
\end{abstract}

PALABRAS CLAVE: Fracturas mandibulares conminutadas, tutores de fijación externa, proyectil balístico.

INTRODUCCIÓN

Las fracturas mandibulares conminutadas se definen como una solución de continuidad en múltiples fragmentos óseos (Al-Assaf \& Maki, 2007). Generalmente son el resultado de un impacto significativo en un área localizada de la mandíbula por traumatismos de alta energía cinética (traumatismos por impacto de proyectil balístico o accidentes de tránsito) (Kim et al., 2016; Carvalho et al., 2019). Su incidencia varía entre un $5 \%$ a un $7 \%$ de las fracturas mandibulares (Alpert et al., 2009). Se caracterizan por presentar un gran daño a nivel de periostio, plano muscular y mucoso, aumentando así la incidencia de la no unión de los fragmentos óseos y posibles infecciones (Wojcik et al., 2016). La ausencia de un tratamiento oportuno puede desencadenar en necrosis de los fragmentos óseos y/o tejidos blandos circundantes (Gibbons et al., 2011).
El tratamiento de las fracturas mandibulares conminutadas por impacto de proyectil balístico, se inicia con el manejo y protección de la vía aérea, seguido de una adecuada hemostasia y aseo quirúrgico de la zona afectada (Mardones-Muñoz et al., 2018). Posterior a la estabilización del paciente, se procede a dar tratamiento quirúrgico de estas fracturas conminutadas. Existen diversas técnicas quirúrgicas, entre ellas la reducción cerrada con fijación externa y la reducción abierta con alambres de acero o con placas y tornillos de osteosíntesis (Ellis 3rd et al., 2003; Carvalho et al.).

Tradicionalmente la técnica más utilizada ha sido la fijación externa por medio del uso de tutores externos mandibulares que proporciona una fijación semirrígida para los segmentos fracturados

\footnotetext{
${ }^{1}$ Equipo Cirugía Maxilofacial, Servicio de Cirugía, Hospital San José, Santiago, Chile.

${ }^{2}$ Departamento de Cirugía y Traumatología Bucal y Maxilofacial, Facultad Odontología, Universidad de Chile, Santiago, Chile.

${ }^{3}$ Departamento de Cirugía Maxilofacial Clínica Las Condes, Santiago, Chile.
} 
MARDONES, M. M.; TORRES, M. C.; GUNCKEL, M. R. \& BRAVO, A. R. Fracturas mandibulares conminutadas por impacto de proyectil balístico: Resultados clínicos de tratamiento con método de fijación alternativo. Int. J. Odontostomat., 15(2):363-369, 2021.

(Cornelius et al., 2009; Chao et al., 2017). En teoría, al tratar estas fracturas de forma cerrada, se mantiene la viabilidad de los fragmentos sin interrumpir su suministro de sangre (Braidy \& Ziccardi, 2009; Wojcik et al.).

En el presente trabajo se reportan los resultados clínicos post operatorios obtenidos en pacientes que fueron tratados con tutor externo mandibular alternativo, confeccionado manualmente durante el tiempo intraoperatorio.

\section{MATERIAL Y MÉTODO}

Se realizó una búsqueda en la base de datos electrónica del hospital de los pacientes diagnosticados con fractura mandibular a través de la plataforma virtual del hospital y el Sistema de Información de la Red Asistencial (SIDRA).

Para llevar a cabo este estudio de presentación de serie de casos de tipo descriptivo, los pacientes firmaron un consentimiento informado previo al examen clínico, registro de datos y fotos clínicas.

La población de estudio correspondió a tres pacientes con diagnóstico de fractura mandibular conminutada tratados mediante fijación con tutor externo mandibular alternativo, operados en los años 2011, 2012 y 2019 respectivamente. Se incluyeron los pacientes que cumplían los criterios de inclusión de diagnóstico de fractura mandibular conminutada, etiología por impacto de proyectil balístico, previamente sometidos a tratamiento de tutores externos mandibulares alternativo con una antigüedad mínima de 1 año, los que fueron retirados con anterioridad a la realización del estudio. Fueron excluidos aquellos pacientes que presentaban fracturas patológicas de la mandíbula asociada a osteomielitis, osteoradionecrosis, quistes o tumores extensos, los tratamientos de fracturas mandibulares conminutadas con reducción abierta y fijación a placas y tornillos de osteosíntesis y los pacientes en tratamiento con tutores externos como método de fijación temporal de sus segmentos óseos fracturados en espera de un tratamiento de reconstrucción posterior.

Todas las cirugías fueron realizadas por el mismo equipo quirúrgico. La técnica empleada se reali- za bajo anestesia general e intubación nasotraqueal. Se efectúa una pequeña incisión en piel a nivel mandibular, en la región que se precisa instalar la aguja Kirschner. Se separan los tejidos blandos por plano hasta llegar al plano óseo y se procede a la instalación de la aguja perpendicular a la cortical externa del fragmento óseo, con el taladro percutor, teniendo precaución de no dañar las estructuras nobles. El hueso mandibular es perforado por las agujas de forma bicortical para lograr la estabilidad de los fragmentos. Se instala un mínimo de 3 agujas Kirschner por segmento. Luego se adapta el tubo de silicona a las agujas instaladas por medio de pequeños cortes para ser atravesado por ellas. Se prepara acrílico de autopolimerización en estado fluido, y rápidamente se vacía en una jeringa de 60cc para ser inyectado al tubo de silicona en toda su extensión. Se mantiene el tubo en posición hasta la polimerización completa y final del acrílico, se eliminan los excesos y se irriga profusamente con suero fisiológico o se aplican compresas húmedas frías para contrarrestar la reacción exotérmica del acrílico. Una vez solidificado el acrílico, se procede a cortar los extremos de las agujas con cizalla de acero y se cubren con resina para evitar las molestias del paciente.

A las 12 semanas post operatorias, los pacientes son controlados clínica e imagenológicamente para evidenciar la remodelación y consolidación ósea de los fragmentos mandibulares e indicar el retiro de los tutores externos. Para su retiro, bajo anestesia local, se procede a cortar las agujas de Kirschner con un disco de acero y posteriormente traccionarlas cuidadosamente con un alicate.

Los tres pacientes que fueron operados en los años 2011, 2012 y 2019, fueron citados para solicitarles una radiografía panorámica y tomografía computarizada (TC) actualizadas para posteriormente realizar el control clínico e imagenológico de los resultados de la técnica de fijación con tutores externos mandibulares.

Durante el control clínico los pacientes fueron evaluados por un observador calibrado para registrar los siguientes hallazgos clínicos post operatorios: dinámica mandibular, presencia o ausencia de pérdida de sensibilidad neurológica en la región mandibular, dolor, movilidad de segmentos óseos, exposición de fragmentos óseos a nivel intraoral, consolidación de fragmentos óseos, oclusión y cicatriz hipertrófica facial. 
MARDONES, M. M.; TORRES, M. C.; GUNCKEL, M. R. \& BRAVO, A. R. Fracturas mandibulares conminutadas por impacto de proyectil balístico: Resultados clínicos de tratamiento con método de fijación alternativo. Int. J. Odontostomat., 15(2):363-369, 2021.

\section{RESULTADOS}

Los pacientes examinados fueron operados durante los años 2011, 2012 y 2019 respectivamente. Todos pertenecían al género masculino, con un promedio de 31 años de edad (Tabla I).

Respecto a los hallazgos post quirúrgicos encontrados, todos los pacientes presentaron una dinámica mandibular favorable con un promedio de apertura acti- va de $44,6 \mathrm{~mm}$, apertura pasiva de $38,7 \mathrm{~mm}$, sin limitación en los movimientos de lateralidad (Tabla II).

Dos pacientes reportaron hipoestesia mentoniana, que correspondió a los pacientes operados en el año 2011 y 2019 respectivamente. Todos los pacientes examinados no presentaron dolor al realizar movimientos funcionales.

Tabla I. Resultados demográficos.

\begin{tabular}{lcccc} 
& Paciente 1 & Paciente 2 & Paciente 3 & Promedio \\
\cline { 2 - 5 } Sexo & Masculino & Masculino & Masculino & - \\
Edad de operación & 33 años & 19 años & 41 años & 31 años \\
Año de operación & 2011 & 2012 & 2019 & - \\
\hline
\end{tabular}

Tabla II. Dinámica mandibular.

\begin{tabular}{lcccc}
\hline & Paciente 1 & Paciente 2 & Paciente 3 & Promedio \\
\hline Apertura cavidad oral activa & $46,8 \mathrm{~mm}$ & $44,8 \mathrm{~mm}$ & $42,4 \mathrm{~mm}$ & $44,6 \mathrm{~mm}$ \\
Apertura cavidad oral pasiva & $35,4 \mathrm{~mm}$ & $38,7 \mathrm{~mm}$ & $42 \mathrm{~mm}$ & $38,7 \mathrm{~mm}$ \\
Lateralidad & Sin limitación & Sin limitación & Sin limitación & - \\
\hline
\end{tabular}
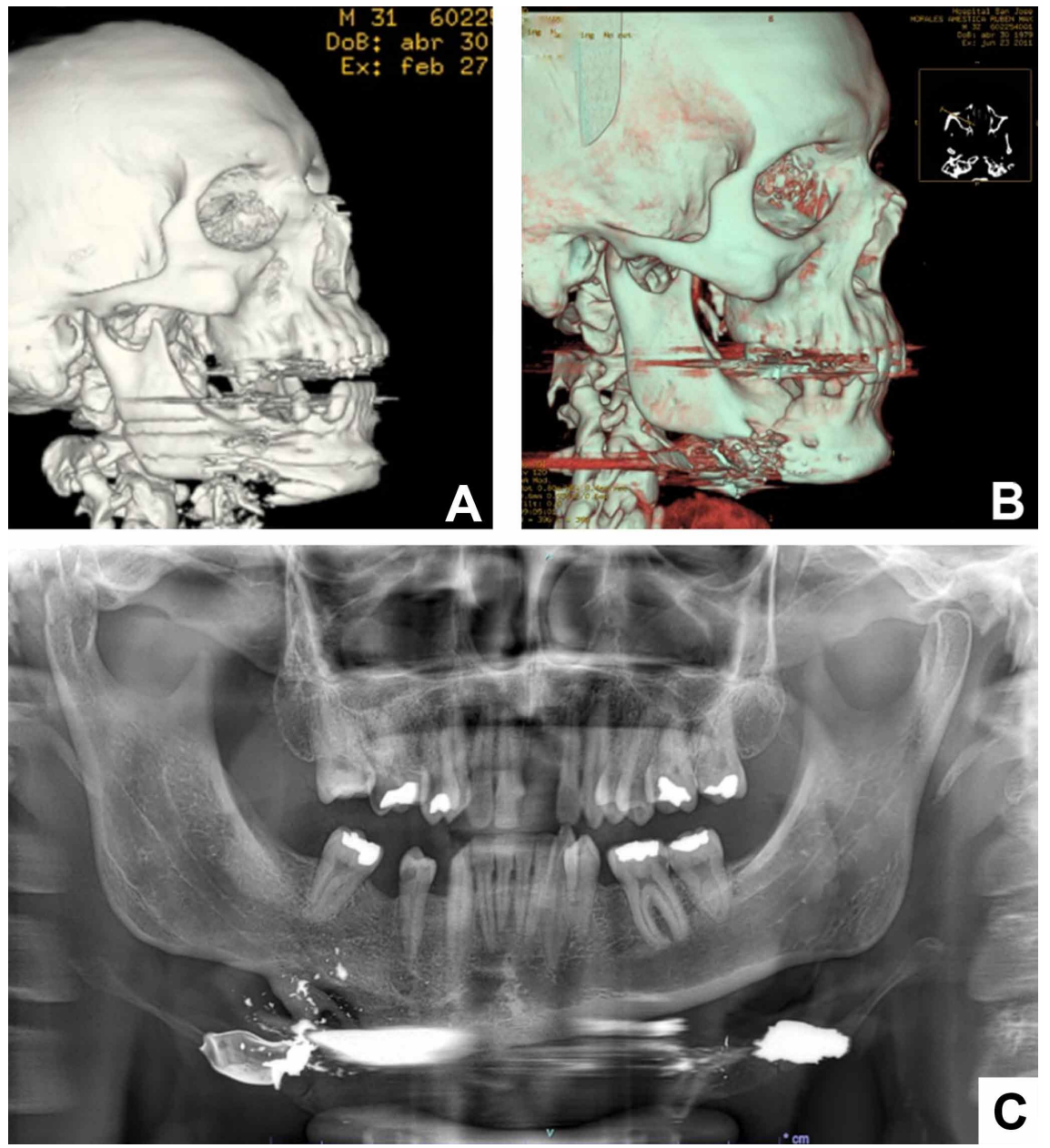

Fig. 1. Paciente 1. (A) TC reconstrucción 3D pre quirúrgico. (B) TC reconstrucción 3D de fractura mandibular consolidada 4 meses posterior a tratamiento con tutor externo alternativo. (C) Radiografía panorámica 9 años posterior a tratamiento con tutor externo alternativo (persiste distorsión de imagen por elementos metálicos del proyectil). 

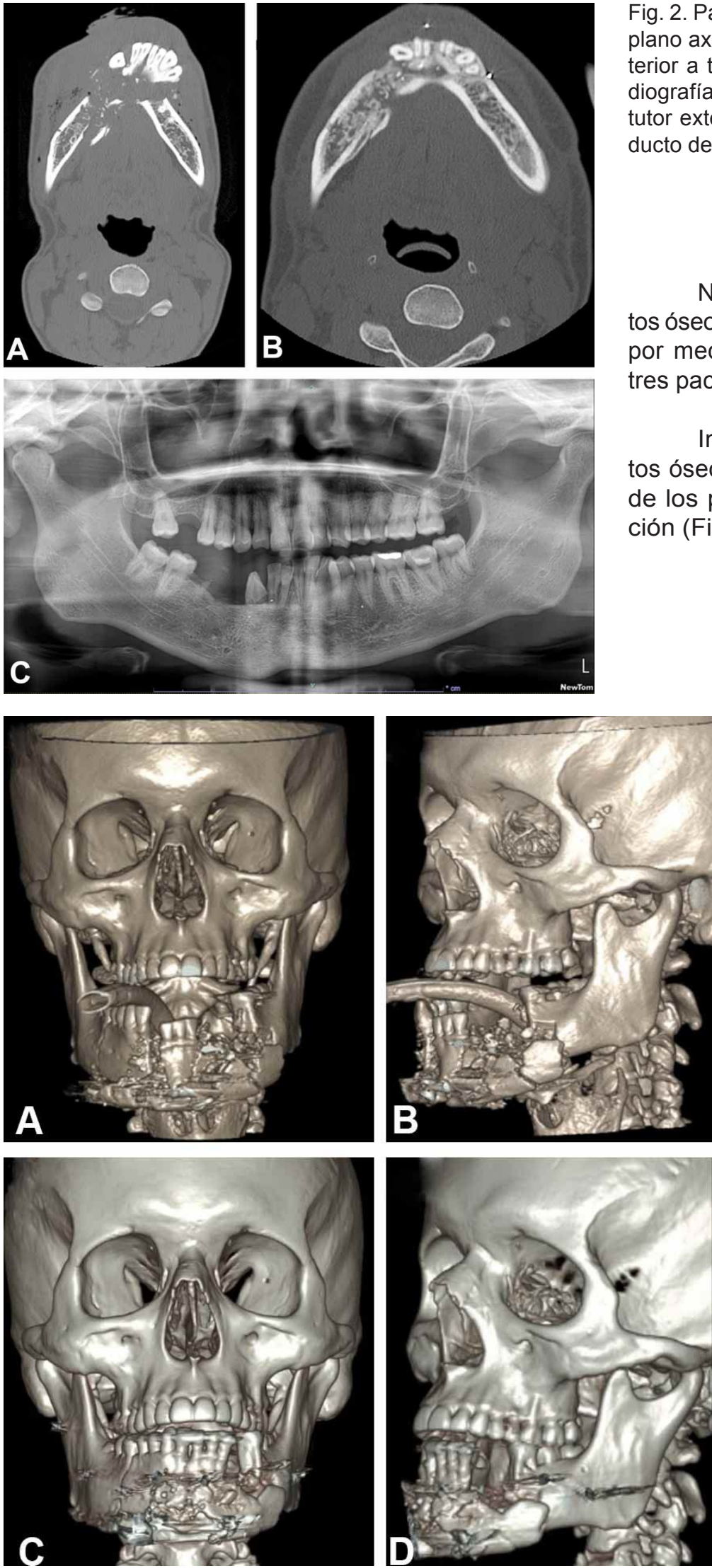

Fig. 2. Paciente 2. (A) TC plano axial pre quirúrgico. (B) TC plano axial fractura mandibular consolidada 5 meses posterior a tratamiento con tutor externo alternativo. (C) Radiografía panorámica 8 años posterior a tratamiento con tutor externo alternativo. Nótese la continuidad ósea producto de la cicatrización del hueso

No se reportó movilidad clínica de los fragmentos óseos tratados, y se verificó su consolidación ósea por medio de radiografía panorámica y TC, en los tres pacientes (Figs. 1, 2 y 3 ).

Intraoralmente, no se observaron fragmentos óseos expuestos a la cavidad bucal en ninguno de los pacientes examinados, ni signos de infección (Fig. 4).
Fig. 3. Paciente 3. (A) y (B) TC reconstrucción 3D pre quirúrgico de fractura mandibular conminutada plano frontal y sagital. (C) y (D) TC reconstrucción 3D 3 meses posterior a tratamiento con tutor externo alternativo, plano frontal y sagital. Se observan imágenes de cicatrización y remodelación ósea. 

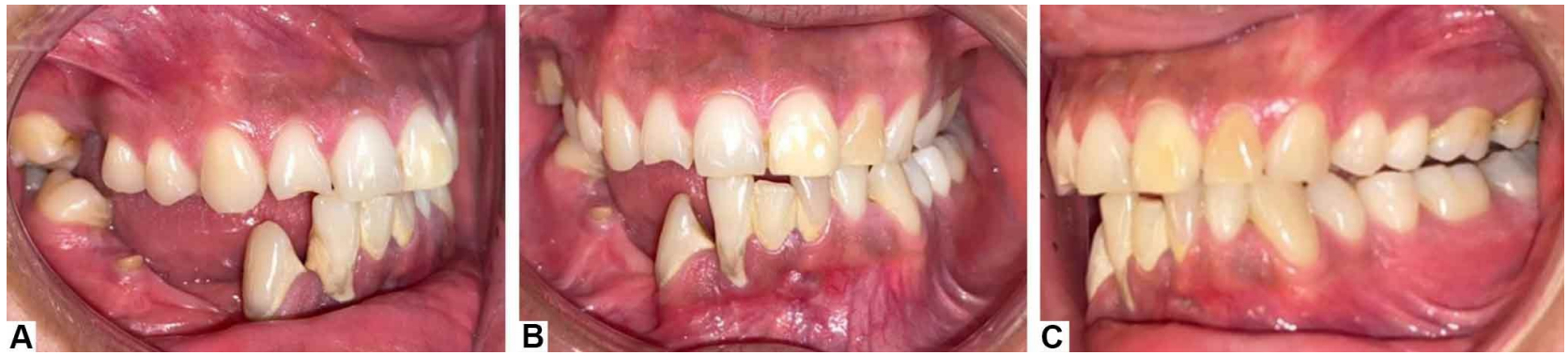

Fig. 4. Imágenes intraorales 8 años posterior a tratamiento con tutor externo alternativo, de paciente 2. Se observa adecuada cicatrización de tejidos blandos y la oclusión resultante posterior al traumatismo.
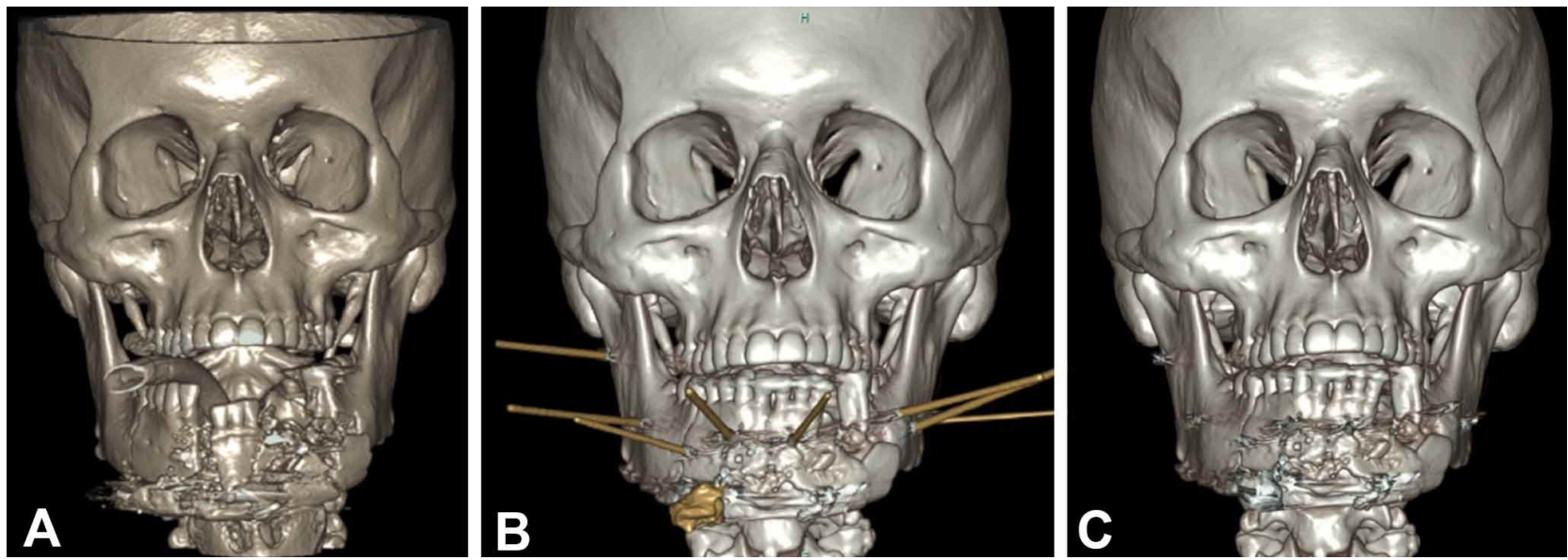

Fig. 5. TC reconstrucción 3D de evolución de tratamiento con tutor externo mandibular alternativo. A. Fractura conminuta B. Fractura fijada con tutor externo C. Remodelación y cicatrización ósea posterior al retiro del tutor externo.

Con respecto al estado de la oclusión, un paciente presentó mordida abierta anterior, quien fue operado con 1 año de antigüedad. Los otros dos pacientes, que fueron operados con una antigüedad mínima de 5 años, reportaron una oclusión estable.

No se presentaron cicatrices hipertróficas a nivel facial (Tabla III).

\section{DISCUSIÓN}

El objetivo de este trabajo es presentar los re- sultados clínicos obtenidos en pacientes con diagnóstico de fractura mandibular conminutada por trauma por impacto de proyectil balístico, tratados por fijación con tutores externos mandibulares alternativo.

Las fracturas conminutadas provocan un daño significativo en el periostio, el músculo y la mucosa, afectando el suministro vascular necesario para la cicatrización ósea (Kazi et al., 2019). En los casos de fracturas conminuta inducidas por un traumatismo de alta velocidad como las heridas por impacto de proyectil balístico, se produce una necrosis inmediata y progresiva de los fragmentos óseos y los tejidos blandos circundantes (Wojcik et al.; Carvalho et al.).

Tabla III. Hallazgos clínicos post operatorios.

\begin{tabular}{lccc}
\hline & Paciente 1 & Paciente 2 & Paciente 3 \\
\hline Sensibilidad neurológica mandibular & Hipoestesia mentoniana & Sin alteración & Hipoestesia mentoniana \\
Dolor & Ausente & Ausente & Ausente \\
Movilidad segmentos óseos & Ausente & Ausente & Ausente \\
Consolidación fragmentos óseos & Presente & Presente & Presente \\
Exposición fragmentos óseos intraoral & Ausente & Ausente & Ausente \\
Oclusión & Estable & Estable & Mordida abierta anterior \\
Cicatriz hipertrófica & Ausente & Ausente & Ausente \\
\hline
\end{tabular}


MARDONES, M. M.; TORRES, M. C.; GUNCKEL, M. R. \& BRAVO, A. R. Fracturas mandibulares conminutadas por impacto de proyectil balístico: Resultados clínicos de tratamiento con método de fijación alternativo. Int. J. Odontostomat., 15(2):363-369, 2021.

La fijación con tutores externos mandibulares es una técnica que manipula los segmentos óseos en su lugar mediante agujas de Kirschner y luego se fijan con algún tipo de conector. A menudo se considera un subtipo de reducción cerrada que proporciona una fijación semirrígida a los segmentos fracturados de la mandíbula (Braidy \& Ziccardi). Este tratamiento permite estabilizar los segmentos mandibulares proximales, favoreciendo una reconstrucción más precisa del segmento afectado, lo que se traduce en una mejor proyección y contorno facial (Jaquet et al., 2011). El objetivo del tratamiento es mantener la relación espacial de los fragmentos mandibulares fracturados hasta su cicatrización (Chrcanovic, 2013).

Las principales indicaciones para el uso de tutores externos son la gran pérdida de hueso mandibular por resecciones tumorales, infecciones que conducen al secuestro óseo, conminución y trauma severo con pérdida de sustancia (Alpert et al.; de Alencar et al., 2018; Kazi et al.). Los pacientes tratados presentaron un trauma de alta energía cinética por impacto de proyectil balístico, generando fracturas conminutadas del hueso mandibular y destrucción de tejidos blandos, motivo por el que se indicó el tratamiento de fijación por medio de tutores externos mandibulares.

La instalación de un tutor externo mediante una técnica cerrada no requiere de una amplia extirpación del periostio en el lugar de la fractura, evitando así el compromiso de la irrigación sanguínea a los fragmentos óseos (de Alencar et al.). En las situaciones en que la conminución se combina con un gran daño perióstico, muscular o mucoso, se espera un aumento de la incidencia de no unión de fragmentos óseos e infecciones. De esta manera, cuando se realiza el tratamiento con tutores externos, se mantiene la viabilidad de los fragmentos sin interrumpir el suministro de sangre (Carvalho et al.). La técnica quirúrgica no requiere de una disección quirúrgica extensa, debido a que se realizan incisiones extraorales pequeñas, y el tiempo quirúrgico es reducido (de Alencar et al.; Kazi et al.). Otras ventajas son el bajo costo de los materiales para la confección de los tutores y su facilidad de instalación (Wojcik et al.).

La instalación prolongada de tutores externos puede comprometer el cumplimiento y la satisfacción del paciente (Kazi et al.). Los tutores externos fueron retirados a las 12 semanas post operatorias, tiempo que permitió la reparación y remodelación ósea del hueso mandibular. En la literatura (Cornelius et al.; Carvalho et al.) se han reportado casos tratados con éxito a las 6 semanas y otros a las 16 semanas post operatorias. Es importante indicar que la duración del tratamiento se verá afectada por factores que interfieren directamente en el índice de éxito, como la aceptación del paciente al tratamiento por tratarse de un dispositivo visible instalado en la cara, una higiene oral satisfactoria y la ausencia de un posible foco infeccioso de origen odontológico (Carvalho et al.).

La tasa de complicaciones post operatorias de este tipo de tratamiento es de un $35 \%$ (Braidy \& Ziccardi), entre ellas se mencionan las infecciones post operatorias, celulitis alrededor de las agujas, formación de fístula salival posterior al daño de la glándula parótida, quemaduras en piel generadas por el proceso de polimerización del acrílico, falta de unión entre los segmentos óseos fracturados, maloclusión, lesión del nervio alveolar inferior (Braidy \& Ziccardi; Kazi et al.).

La consolidación de los segmentos óseos se presentó en todos los pacientes, evidenciándose a través de la radiografía panorámica y TC actualizados (Fig. 5). Aunque dos pacientes fueron operados hace más de 5 años y el tercero hace 1 año atrás, no se reportó falta de unión en ninguno de los segmentos ni movilidad clínica asociada a estos. Tampoco se reportó exposición de los fragmentos óseos a nivel intraoral en ninguno de los pacientes, evidenciando una cicatrización favorable sin infección. Con respecto a la oclusión de los pacientes, uno presentó mordida abierta anterior (operado hace 1 año atrás) y los otros dos pacientes presentaron una oclusión estable. Según un estudio de Ellis 3rd et al. reportaron un $23,5 \%$ de pacientes con falta de consolidación de los segmentos óseos, y un $11,7 \%$ de los pacientes desarrollaron una maloclusión.

Todos los pacientes presentaron una dinámica mandibular favorable y sin limitación en los movimientos de lateralidad. Sin embargo, una de las complicaciones reportadas fue la hipoestesia mentoniana en dos de los pacientes controlados.

La instalación de las agujas y su posterior retiro puede generar cicatrices hipertróficas (Braidy \& Ziccardi), noobstante, ninguno de los pacientes controlados presentó cicatrices hipertróficas a nivel facial.

Cornelius et al. afirmaron que este método de fijación sigue siendo una opción de tratamiento valiosa que permite una unión entre las áreas de fractura conminuta o defectos con una calidad ósea comprometida y/o un tejido blando afectado. La fijación por 
MARDONES, M. M.; TORRES, M. C.; GUNCKEL, M. R. \& BRAVO, A. R. Fracturas mandibulares conminutadas por impacto de proyectil balístico: Resultados clínicos de tratamiento con método de fijación alternativo. Int. J. Odontostomat., 15(2):363-369, 2021.

tutores externos mandibulares es una técnica que reportó resultados favorables en los pacientes que fueron tratados, logrando consolidación ósea de los segmentos fracturados, oclusión estable y aceptable dinámica mandibular.

\section{CONCLUSIÓN}

El tratamiento de fracturas mandibulares conminutadas con tutores externos permite una estabilización de los fragmentos óseos, favoreciendo posteriormente una neoformación ósea y remodelación.

En la serie de casos presentada, se reportaron resultados clínicos favorables de consolidación de fragmentos óseos, dinámica mandibular y estabilidad oclusal, sin embargo, se requieren estudios a futuro con una muestra mayor de pacientes para establecer de forma objetiva las ventajas de esta técnica.

MARDONES, M. M.; TORRES, M. C.; GUNCKEL, M. R. \& BRAVO, A. R. Mandibular Fractures Comminuted by Ballistic Projectile Impact: Clinical Results of Treatment with Alternative Fixation Method. Int. J. Odontostomat., 15(2):363369, 2021.

ABSTRACT: Comminuted mandibular fractures from ballistic missile impact are a therapeutic challenge due to the presence of multiple bone fragments and the involvement of periosteum, muscle and mucous membrane. Their initial treatment requires emergency medical and surgical stabilization and later treatment of the fractured bone segments. External fixation with mandibular stakes makes it possible to stabilize the mandibular segments in a closed manner, thus maintaining the viability of the fragments without interrupting their blood supply. The aim of this work is to present the post-surgical results obtained in three clinical cases of patients with diagnosis of comminuted mandibularfracture by ballistic missile impact, treated by an alternative method to the conventional external fixation tutors.

KEY WORDS: Comminuted mandibular fractures, external fixation tutors, ballistic projectile.

\section{REFERENCIAS BIBLIOGRÁFICAS}

Al-Assaf, D. A. \& Maki, M. H. Multiple and comminuted mandibular fractures: treatment outlines in adverse medical conditions in Iraq. J. Craniofac Surg., 18(3):606-12, 2007.

Alpert, B.; Tiwana, P. S. \& Kushner, G. M. Management of comminuted fractures of the mandible. Oral Maxillofac. Surg. Clin. North Am., 21(2):185-92, 2009.
Braidy, H. F. \& Ziccardi, V. B. External fixation for mandible fractures. Atlas Oral Maxillofac. Surg. Clin. North Am., 17(1):45-53, 2009.

Carvalho, P. H. R.; da Hora Sales, P. H.; da Rocha, S. S.; Cavalcanti, A. M. M.; de Jesus Rodrigues Mello, M. \& Menezes Junior, J. M. S. Treatment of comminutive fractures by firearm projectiles with adapted wrist external fixator. Oral Maxillofac. Surg., 23(4):5015, 2019.

Chao, D. M.; Jin, S.; Khatri, K. S.; Long, Z. J.; Lei, T. \& Lisheng, H. Managing a grossly comminuted and infected mandibular fracture using a maxillary extra-oral distractor as stabilizing agent: $\mathrm{A}$ clinical case report. Trauma Case Rep., 9:10-2, 2017.

Chrcanovic, B. R. Open versus closed reduction: comminuted mandibular fractures. Oral Maxillofac. Surg., 17(2):95-104, 2013.

Cornelius, C. P.; Augustin, J. B. \& Sailer, L. K. External pin fixation for stabilization of the mandible--comeback of a method: historical review and first experiences with the 'mandible external fixator'. Oral Maxillofac. Surg., 13(1):1-14, 2009.

de Alencar, M. G. M.; de Bortoli, M. M.; da Silva, T. C. G.; de Oliveira e Silva, E. D. \& Laureano Filho, J. R. Suitability of wrist external fixator for treatment of mandibular fracture. J. Craniofac. Surg., 29(4):e371-2, 2018

Ellis 3rd, E.; Muniz, O. \& Anand, K. Treatment considerations for comminuted mandibular fractures. J. Oral Maxillofac. Surg., 61(8):861-70, 2003.

Gibbons, A. J.; Mackenzie, N. \& Breederveld, R. S. Use of a custom designed external fixator system to treat ballistic injuries to the mandible. Int. J. Oral Maxillofac. Surg., 40(1):103-5, 2011.

Jaquet, Y.; Higgins, K. M. \& Enepekides, D. J. Pin guidance of reconstruction plate contour: an expanded role of external fixation. Laryngoscope, 121(9):1896-8, 2011.

Kazi, A. A.; Lee, T. S.; Vincent, A.; Sokoya, M.; Sheen, D. \& Ducic, Y. The role of external fixation in trauma and reconstruction of the mandible in the age of rigid fixation. Facial Plast. Surg., 35(6):61422, 2019.

Kim, J.; Choi, J. H.; Chung, Y. K. \& Kim, S. W. Panfacial bone fracture and medial to lateral approach. Arch. Craniofac. Surg., 17(4):181-5, 2016.

Mardones-Muñoz, M.; Bravo-Ahumada, R.; Altschiller-Mardones, J. \& Pérez-Gutiérrez, H. Mandibular fractures comminuted by ballistic projectile impact: initial treatment proposal using alternative fixation method. Int. J. Odontostomat., 12(4):423-30, 2018.

Wojcik, T.; Nicot, R.; Ferri, J. \& Raoul, G. A cheap hand-made mandibular external fixator? J. Craniofac. Surg., 27(7):1839-41, 2016.

Dirección para correspondencia:

Dra. Coral Torres Manríquez

Cirujana Dentista

Equipo Cirugía Maxilofacial

Servicio de Cirugía

Hospital San José

Santiago

CHILE

E-mail: torresm.coral@gmail.com 